\title{
Human papilloma viruses and cervical tumours: mapping of integration sites and analysis of adjacent cellular sequences Eugene Klimov ${ }^{1}$, Svetlana Vinokourova ${ }^{2,4}$, Elena Moisjak ${ }^{1}$, Elian Rakhmanaliev1, Vera Kobseva², Laimonis Laimins ${ }^{3}$, Fjodor Kisseljov² and Galina Sulimova*1
}

Address: ${ }^{1}$ N.I. Vavilov Institute of General Genetics, RAS, Moscow, Russia, 2 Institute of Carcenogenesis, Cancer Research Center, Moscow, Russia, ${ }^{3}$ Northwestern University, Chicago, USA, ${ }^{4}$ Division of Molecular Pathology, University of Hedelberg, Im Neuenheimer Feld 110, 69120 Heidelberg, Germany and ${ }^{5}$ These authors contributed equally to this work

E-mail: Eugene Klimov - klimov_eugeney@mail.ru; Svetlana Vinokourova - Vinokourova@mail.ru; Elena Moisjak - plena@freemail.ru; Elian Rakhmanaliev - elian-rr@newmail.ru; Vera Kobseva - vkobzeva@mail.ru; Laimonis Laimins - l-laimins@northwestern.edu; Fjodor Kisseljov - f_kiss@crc.umos.ru; Galina Sulimova* - sge@vigg.ru

${ }^{*}$ Corresponding author †Equal contributors

Published: 13 October 2002

BMC Cancer 2002, 2:24

This article is available from: http://www.biomedcentral.com/I47I-2407/2/24

(C) 2002 Klimov et al; licensee BioMed Central Ltd. This article is published in Open Access: verbatim copying and redistribution of this article are permitted in all media for any purpose, provided this notice is preserved along with the article's original URL.
Received: 17 June 2002

Accepted: 13 October 2002

\begin{abstract}
Background: In cervical tumours the integration of human papilloma viruses (HPV) transcripts often results in the generation of transcripts that consist of hybrids of viral and cellular sequences. Mapping data using a variety of techniques has demonstrated that HPV integration occurred without obvious specificity into human genome. However, these techniques could not demonstrate whether integration resulted in the generation of transcripts encoding viral or viral-cellular sequences. The aim of this work was to map the integration sites of HPV DNA and to analyse the adjacent cellular sequences.
\end{abstract}

Methods: Amplification of the INTs was done by the APOT technique. The APOT products were sequenced according to standard protocols. The analysis of the sequences was performed using BLASTN program and public databases. To localise the INTs PCR-based screening of GeneBridge4RH-panel was used.

Results: Twelve cellular sequences adjacent to integrated HPVI6 (INT markers) expressed in squamous cell cervical carcinomas were isolated. For I I INT markers homologous human genomic sequences were readily identified and 9 of these showed significant homologies to known genes/ ESTs. Using the known locations of homologous cDNAs and the RH-mapping techniques, mapping studies showed that the INTs are distributed among different human chromosomes for each tumour sample and are located in regions with the high levels of expression.

Conclusions: Integration of HPV genomes occurs into the different human chromosomes but into regions that contain highly transcribed genes. One interpretation of these studies is that integration of HPV occurs into decondensed regions, which are more accessible for integration of foreign DNA. 


\section{Background}

Cervical cancer is the second most common cause of cancer related mortality in women world-wide. Most cervical cancers are squamous cell carcinomas that develop through a distinct pattern of morphological progression. Cervical tumours are associated at a high frequency with infection by human papilloma viruses (HPV) and sequences of the so-called high risk HPV (types 16 and 18 and related) are detected in nearly every tumour examined [1]. Viral DNA persists in tumour cells in episomal and/or integrative forms with the retention of the two of viral transforming genes (E6 and E7) in all tumours analysed. The expression of viral sequences is controlled by sequences within the upstream regulatory region (URR) that is located upstream of the E6 gene. Viral transcripts include a variety of spliced RNA. In cases where the episomal form of HPV predominates, the full expression of E6 and E7 genes occurs (few splicing forms), while in the case of integrative form - the expression of cellular sequences downstream to 3 ' of viral sequences also can be detected in the form of fused viral-cellular RNAs [2]. It is also possible that, in some cases, after integration viral sequences become "silent" [3,4].

Analysis of integration sites based on different techniques and their mapping in the human genome revealed that DNA integration of different HPV types occurred in different chromosomal sites without visible specificity [5-12]. It was demonstrated that in some case sites of viral genome integration mapped to regions of human genome that often underwent chromosomal rearrangements and deletions. In other cases HPV integration sites were mapped to so called fragile sites, or in regions where genes that are directly or indirectly involved in the control of cell proliferation have been localised. Not all of these methods give precise and adequate data and in addition they can not discriminate between "silent" and integrated viral DNA. Furthermore, in many cases these methods do not allow precise physical mapping of integrative viral sequences. The use of the so-called APOT techniques have greatly simplified the analysis of expressed integration sites and allowed characterisation of a large number of integration sites through analysis of expressed joint viralcellular sequences. The major conclusion from these studies was that integration is non-specific [12].

Although analysis of many integration sites has already been described, a detailed examination using different techniques seemed important, since it provides additional information concerning the interaction of viral and host genomes and the role of this process in genetic program of cancer cell.

The primary aim of this work was the physical mapping of the integration sites of HPV 16 DNA in chromosomes of human cervical squamous cell carcinomas by isolation of integration sites by APOT technology. This was followed by mapping of the expressed virus-cellular sequences generated by integration using PCR screening of a panel of radiation hybrids of somatic cells as well as database analysis of cellular sequences located adjacent to integration sites.

\section{Methods \\ Original materials}

All tumour samples were collected during surgery in the clinics of Cancer Research Centre (Moscow) and were kept frozen in liquid nitrogen. DNA and RNA isolation and HPV typing were performed according to techniques described earlier [13]. Only squamous cell carcinomas containing HPV 16 sequences were analysed.

\section{Amplification of fusion transcripts}

Human genomic sequences adjacent to integrated HPV 16 DNA from squamous cell cervical carcinomas have been isolated with the use of the APOT technique [2] based on reverse transcription with further two step amplification of RT-product [3,2] (HPV integration sites - INT). This technique was described earlier in detail $[2,3]$.

Briefly reverse transcription was performed using an adapter linked oligo(dT)-primer [14] followed by seminested PCR using E7-specific 5' primed primers and specific oligo(dT)/adapter primers (3' primed) [2,12,3]. PCR products were transferred onto nylon membranes and hybridised with HPV E7 and E4 specific probes to discriminate episomal from integration derived transcripts. PCR products containing integration sites were excised from the gel and extracted using the QIAGEN Gel extraction kit (Qiagen, Hilden, Germany). Sequencing reactions were performed using Big-Dye terminator DNA-sequencing Kit (Perkin-Elmer, Boston, USA) and an ABI Prism $310 \mathrm{Ge}$ netic analyzer (Applied Biosystems, Foster City, USA). Sequencing results were analysed using BLASTN-program provided by the National Cancer Insitute. The sequences of the 12 studied INTs were submitted in EMBL Database (AccN: AJ431608 - INT254, AJ431609 - INT259, A431610 - INT431, AJ431611 - INT407, AJ631612 INT290, AJ431614 - INT505, AJ431615 - INT477, AJ431616 - INT467, AJ431617 - INT466, AJ431618 INT421, AJ431619 - INT475, AJ431620 - INT423).

Physical mapping of HPV I 6 DNA on human chromosomes To localise INTs, PCR-based screening of a somatic cell radiation hybrid (human/hamster) GeneBridge4-RH-panel (Research Genetics Inc., USA) was applied as described earlier [15].

PCR was performed in $12.5 \mu$ reactions, using a PTC$100^{\mathrm{TM}}$ thermocycler (MJ Research Inc., USA). Some modi- 
Table I: Primers and PCR conditions

\begin{tabular}{|c|c|c|c|}
\hline Marker & Primers $3^{\prime}-5^{\prime}$ & Annealing $\mathrm{T}^{\circ} \mathrm{C} /$ time, sec & Amplification product, bp \\
\hline \multirow[t]{2}{*}{ INT254 } & F: CTTTCAGCAAAGGATGTCATTG & $57^{\circ} \mathrm{C} / 50$ & 203 \\
\hline & R: GCAAGGCTTATTAAGCTTCAGC & & \\
\hline \multirow[t]{2}{*}{ INT259 } & F: ATCTACAGACAGCATGGTACGG & $60^{\circ} \mathrm{C} / 45$ & 107 \\
\hline & R: TCAGAACACAACAAAAGGATGC & & \\
\hline \multirow[t]{2}{*}{ INT43I } & F: TCCAAAAAAATGAGGCAATCTC & $60^{\circ} \mathrm{C} / 50$ & 101 \\
\hline & R: GGACTGAGGGAATAAACGACAC & & \\
\hline \multirow[t]{2}{*}{ INT407 } & F: AGCAGGAGATGACAAGATGTTG & $55^{\circ} \mathrm{C} / 35$ & 103 \\
\hline & R: CACAAGTAGCTGAGACCACAGG & & \\
\hline \multirow[t]{2}{*}{ INT290 } & F: AAGTTCCACCATTCAAGACCAG & $55,5^{\circ} \mathrm{C} / 45$ & 215 \\
\hline & R: TCATGATATCCTTGGTGTCCTG & & \\
\hline
\end{tabular}

fications were introduced, including the "hot start" technique, as recommended in the instructions for Maxi$\mathrm{Taq}^{\mathrm{TM}}$, manufactured by Biokom Inc. (Russia). The amount of DNA per tube was 8-10 ng. The nucleotide sequences of the primers used in these studies are presented in Table 1. Conditions for annealing, priming, and other parameters were optimised using total human and hamster DNAs (Table 1). The analysis of PCR products using agarose and polyacrylamide gels was performed following standard protocols.

The results of PCR screening of the RH-panel were analysed with RHMAPPER through the server of the Whitehead Institute/MIT Centre for Genome Research [http:// carbon.wi.mit.edu:8000/cgi-bin/contig/rhmapper.pl]. To reconcile these data with previously reported information regarding maps of the chromosome, three additional sources were used [http://www.ncbi.nlm.nih.gov/]; [http://www.stanford.edu/RH/]; [http://www.tigr.org/].

\section{Search for sequences homologous to cellular markers of DNA HPV 16 integration sites}

Search for homologies was carried out using the BLASTN program [http://www.ncbi.nlm.nih.gov/BLAST/], and databases GeneBank and EMBL. This search was done as follows:

a) search for homologies of INT sequences with human genomic sequences in HGMT database;

b) selection of drawling genomic sequences, which include sequences homologous to INT markers;

c) selection of genomic fragments, that flank INT marker from 5' and 3' ends, as long as 2000 nucleotides from both ends; d) search for homologies of genomic DNA fragments selected with genes and cDNAs by use of well known databases (GeneBank and EMBL).

Information concerning proteins, encoded by genes that are homologous to DNA HPV 16 integration sites and are mapped in their site of localisation, was obtained from OMIM database [http:///www.ncbi.nlm.nih.gov/] and LocusLink [http://www.ncbi.nlm.nih.gov/LocusLink/].

The data on RH-mapping are presented in March 2001 and the data on mapping of markers on chromosomes and genomic contigs - to September 2001. Information about search of homologies corresponds to the public databases for January 2002.

\section{Results}

\section{Detection of DNA HPV I 6 integration sites}

APOT technique was used to isolate human genomic sequences adjacent to integrated HPV 16 DNA (HPV integration sites - INTs) expressed in squamous cell cervical carcinomas. This technique is based on a modification of RT-PCR methods using specific primers, one of which was localised in E7 region of viral genome and a second one which contains specific adapter sequences joined with oligo(dT) $[2,3]$. As a result we generated fusion transcripts that encompassed parts of the human genomic DNA. Some of the transcripts may be spliced into non-coding sequences within the 3' untranslated part of the respective genomic mRNAs and others - into intronic regions close to the transcription initiation site of the respective gene as was recently shown by Wentzensen et al. [12]. We have not analysed in detail these types of fusion transcripts, but in the all cases our methods allowed discrimination between expressed episomal and integrative forms of HPV DNA. 
Twelve INT sequences have been characterised from different individual squamous cell cervical carcinomas. The length of the cellular sequences containing polyA tails and fused to viral sequences varied from 140 to 450 nucleotides. The sequences were submitted in EMBL Database (see Methods section for AccN) and used for searching homologies to human genomic sequences, genes and ESTs. Corresponding primers were created to these cellular sequences at integration sites and were used for screening of RH-panel of somatic cell hybrids (Table 1).

\section{Analysis of nucleotide sequences homologous to integra- tion sites}

Searches for human nucleotide sequences that were homologous to INT markers were carried out using BLASTN program and public databases as described in Materials and Methods. Homologies greater than $90 \%$ for sequences not less than 100 nucleotides in size were considered significant.

Homologies with cDNA clones were found for only 5 of the 12 analysed INT clones and this may in part be due to the relatively small size of the markers examined (150350 base pairs). Homologous genomic sequences were found for 11 INT markers (Table 2, see Additional file 1). This allowed us to extend significantly the search for EST homologies to longer cellular sequences (up to $2.000 \mathrm{bp}$ ) that flanked integrated viral genome. For almost all markers (excluding INT431, INT254 and INT407) homologies with cDNAs that are highly similar to cDNAs for known genes or with non-identified cDNAs were found.

In the cases where homology with the same gene or EST was detected both with INT markers and with adjacent cellular sequences, one may conclude that (markers INT290, INT505, INT466 and INT423) HPV DNA had integrated into a human gene in this cervical carcinoma. The integration of viral DNA occurred into the terminal exon of GLS gene and provides a clear example of integration into an actively transcribed gene (marker INT423).

An integration site marked as INT466 is of special interest as it is highly likely that viral DNA is incorporated into the exon 5 of the interferon/beta receptor like gene (LOC152028). One part of the marker is homologous to the exon 5 and the other part is homologous to the exon 6 of this gene.

Marker INT290 was found to be homologous to two genes: to the WASF2 gene and the gene for the protein similar to WASF2 protein (LOC158537) located on the different chromosomes (1p36.11-34.3 and Xp11.3 accordingly).
The corresponding human genomic sequence was not found for one of the markers (INT467) although a high level of homology was found with a cDNA similar to mRNA of multicopy gene 40S ribosomal protein S27 (MPS1), which is localised on several human chromosomes $(1,2,3,4,5,6,7,11,12,15,18,19)$.

In four cases homologies were not found between INT markers (INT259, INT477, INT421 and INT475) and any genes or ESTs. But the sequences flanking these markers from 5'- or 3'-ends were homologous to ESTs (Table 2 see Additional file 1). For three INT markers (INT254, INT407 and INT431) and their adjacent cellular sequences homologies to genes or ESTs were not found. For this reason, the physical locations of these markers were determined using RH-mapping. All variants of cDNAs that are homologues to INT markers and flanking genomic sequences are present at high levels in expression databases (10-50 clones) indicating a high level of expression. For this study, clones were obtained from different human normal organs and tissues, as well as from different pathologies (including tumours).

\section{RH-mapping of INT markers}

The known locations of the cDNAs and human genomic sequences identified above allowed us to localise nine INT markers, which are highly homologous to these cDNAs (Table 2, see Additional file 1). Two INT markers were found to have multiple chromosomal localisation (INT467 and INT290). In seven cases the locations of the integration sites are specific for each tumour and the markers are present as a single copy in one of the chromosomes. RH-mapping has been used to localise INT254, INT431 and INT407, for which there were no homologies to genes or ESTs and INT290. In addition, the location of the INT259 was also determined using RH-mapping because the lengths of the homologous sequences were too short (104 bp and $59 \mathrm{bp}$ ).

Altogether five INT markers were localised on the radiation hybrid map of Whitehead Institute (WI-RH-Map) as a result of our screening (Table 3, see Additional file 2). To allow for a convenient comparison of our data with the public databases, we converted the INT marker positions in Whitehead Institute RH-map (WI-RH-Map) into the GeneMap99-GB4 (GM99-GB4) (Table 3, see Additional file 2). This recalculation should not generate any errors because the physical locations for the majority of framework markers are known for both RH-maps. This procedure allows for a more complete analysis of information from the public databases. Physical intervals and subloci of chromosomes in which INT sequences are located were determined. YAC-contigs and genomic contigs, to which mapped markers belong, were identified and the physical localisation on chromosomes was defined (Table 3, see 


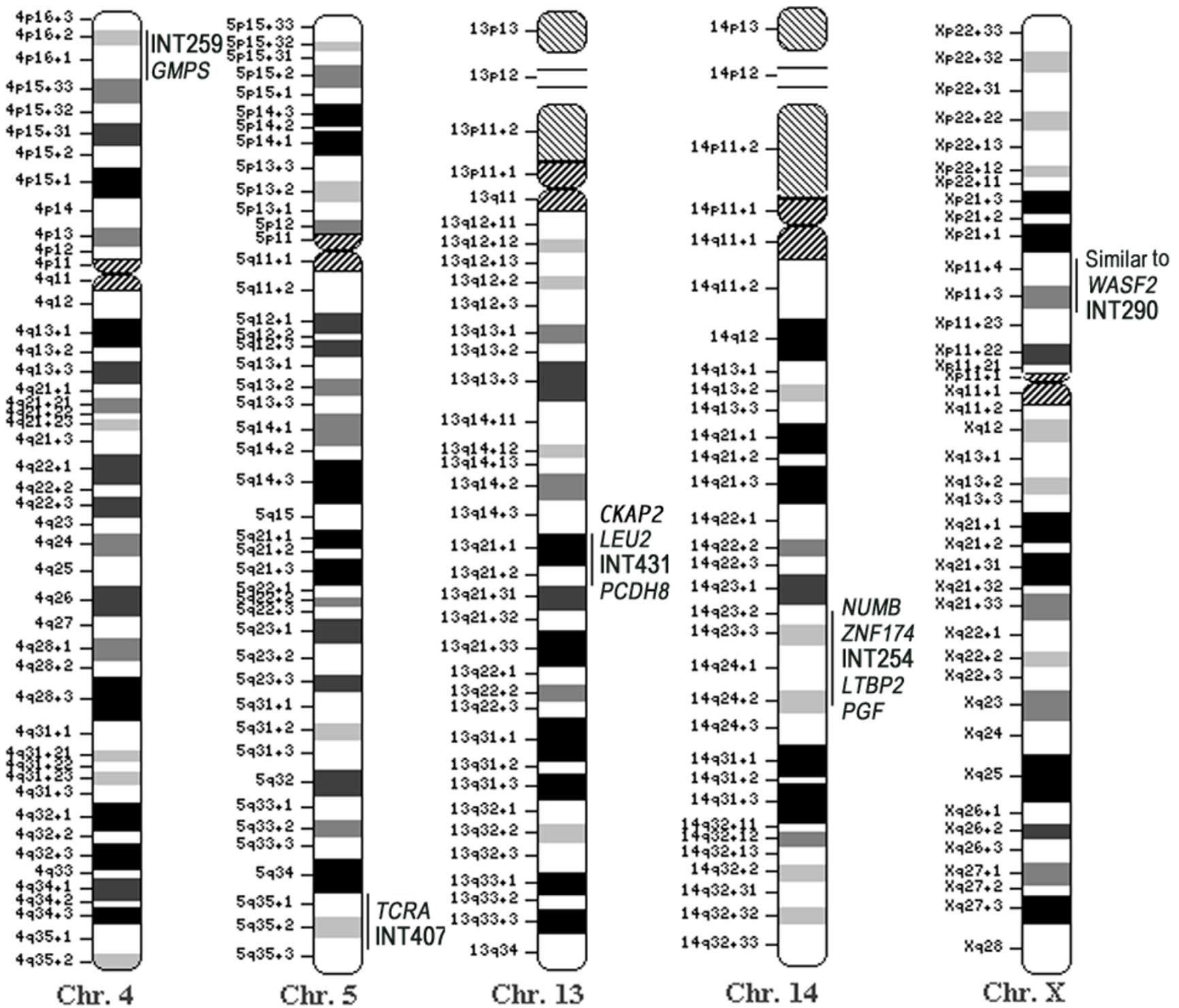

Figure I

Locations of INT markers on human chromosomes (RH-mapping data). Bold marked INT markers. In Italics marked genes that are adjacent to INT markers: similar to WASF2 - Wiskott-Aldrich syndrome gene; ZNFI 74 - zinc finger protein I74; NUMB numb homologue (Drosophila) (neurone grow factor); LTBP2 - latent transforming growth factor beta binding protein 2; PGF placental growth factor; EDG2 - endothelial differentiation, lysophosphatidic acid G-protein-coupled receptor, 2; PCDH8 - protocadherin 8; CTNNALI - catenin (cadherin-associated protein), alpha-like I; TCRA - T cell receptor alpha locus; CKAP2, LEU2 tumour-suppressers genes.

Additional file 2). The physical locations of five INT markers mapped by us were in a good agreement with the locations of the corresponding contigs of human genomic sequences. The locations of these integration sites are also specific for each tumour and all of the markers are present as a single copy in one of the chromosomes including INT290 located on Xp11.21. The maps of chromosomes in which the sites of INT markers and genes located in the same regions of the chromosomes are indicated are presented on Fig. 1.

The analysed HPV integration sites were found to be located on different human chromosomes and no obvious degree of specificity of the integration sites is present. Nevertheless, it is interesting to note that one of INT sites (INT431) mapped in the region 13q14-q21 that is saturat- 
ed with tumour suppressers genes such as CKAP2, LEU1 and CLL-4[16]. Some other genes, that are located around other INT positions, may also belong to the group of tumour suppressers. The genes that participate in the processes of development and differentiation of tissue (NUMB, LTBP2, PGF andEDG2), genes encoding for signal proteins (similar to WASF2, ZNF 174), and proteins of cytoskeleton (PCH8, CTNNAL1) are also present in these loci (Fig. 1).

\section{Discussion}

The analysis of the physical locations of integrated HPV 16 DNA expressed in squamous cell carcinomas of cervix using RH-technology and examination of the cellular sequences adjacent to the INTs allows us to confirm previous studies indicating that viral integration sites are randomly distributed in human genome [11,12]. Integration of viral DNA occurs into different regions of chromosomes and does not seem to be site-specific. Interestingly, many important genes participating in processes of cellular growth and differentiation were found to be located around these sites of viral integration (Fig. 1). These included three markers (INT254, INT431 and INT505) that are present in the area of known fragile sites and mapped to $14 \mathrm{q} 23$ (INT254 - 14q23.2), 13q21.2 (INT431 $13 \mathrm{q} 21.23$ ) and $10 \mathrm{q} 23.3$ or $10 \mathrm{q} 24.2$ (INT505 10q23.32).

Analysis of ESTs homologous to INT sequences allowed us to obtain additional information about the putative genes into which viral DNA is incorporated. As a rule these are sequences are similar to genes encoding for proteins that are important for cell division, differentiation and cell viability. For instance membrane protein myoferlin (gene MYOF - marker INT505) participates in the development and differentiation of muscle tissue [[17]; OMIM: 604603]. Ribosomal protein S27 (marker INT467) contains a predicted zinc finger domain of the C4 type and can bind to DNA [[18]; OMIM: 603702]. The protein responsible for Wiskott-Aldrich syndrome (mRNA similar to WASF2 gene - marker INT290) belongs to the family of GTP-ases that transduce signal to actin from cytoskeleton. It was shown for WASF2 gene, that its expression induces the abnormal accumulation of actin [[19]; OMIM: 605875]. Interferon (marker INT466) belongs to the protein factors that are associated with cellular response to viral infections [OMIM: 107450]. Glutaminase (GLS - marker INT423) participate in the synthesis of glutaminate, which appear to be a neurotransmitter [[20,21]; OMIM: 138280]. Nuclear gene RTN4IP1 (marker INT475) encoded reticulon 4 interacting protein 1, which function is unknown [LocusLink ID: 84816].
All these data are in a good correlation with other recently published studies $[10,11]$. The conclusions of these additional studies, which used other techniques of analysis of integration sites, indicated that the sites of integration may be associated with fragile sites as well as a different spectrum of genes or EST sequences. It is also necessary to point out that we as well as Wentzenzen et al. [12] analysed only expressed sequences from integration sites in which cellular sequences localised downstream to viral sequences.

These data address the question about the possible role of HPV DNA integration in tumour development. Among cervical tumours around 50\% contain viral DNA in episomal form $[3,22,23]$. This may indicate that persistence of viral transforming genes E6 and E7 products that inactivate products of tumour-suppresser genes $p 53$ and $R b 105$ and some of cyclins [24-26] is important and that integration does not play a crucial role in cervical tumour progression.

In our study we also demonstrated that cDNAs, that are homologous to INT markers, have high levels of expression in cells. These data were also confirmed by our additional experiments not presented in this manuscript analysing amplified sequences transcribed from total RNA isolated from different normal and tumour cells of epithelial origin. This suggests that the regions with actively transcribed genes are in decondensed form, forming accessible regions for integration of foreign genetic material. It is possible that viral DNA integration into actively transcribed regions of cellular genome is a safety mechanism to secure of viral genetic information.

\section{Conclusions}

Twelve human genomic sequences adjacent to integrated HPV 16 DNA (HPV integration sites - INTs) expressed in squamous cell cervical carcinomas have been characterised. A BLASTN homology search was performed for the viral DNA integration sites and their surrounding sequences against the HGMT and EST databases. 11 INT markers were found to be homologous human genomic sequences and 9 of them had significant homologies to known genes or ESTs. Locations of the 6 INTs were determined on the basis of the known locations of the corresponding cDNAs. The RH-mapping technique has been used to physically localise of five HPV INTs: INT254, INT431 and INT407 (no homologies to genes or ESTs), INT290 (homologies to two genomic sequences localised on different chromosomes) and INT259 (short length of homologous sequences).

The physical locations of five INT markers mapped by us were in a good agreement with the locations of the corresponding contigs of human genomic sequences. The loca- 
tions of these integration sites are also specific for each tumour and all of the markers are present as a single copy in one of the chromosomes including INT290 located on Xp11.21. All of the INTs (mapped by us and localised using the data of genomic positions of homologous cDNAs) are distributed in the regions with the high level of expression.

Integration of HPV genome occurs into the different human chromosomes but into the regions that contain highly transcribed genes important for cell viability. One possible interpretation of this phenomenon is that the regions with actively transcribed genes are in extended chromatin configuration, forming accessible regions for integration of foreign genetic material. It is also necessary to point out that we analysed only expressed sequences from integration sites in which cellular sequences localised downstream to viral sequences. We cannot exclude the possibility that after integration certain viral sequences become "silent" and this proposal has been confirmed by Kiselev et al [3] and Van Tine et al [4].

\section{Competing interests}

None declared.

\section{Authors' contributions}

Klimov E. carried out the RH-mapping for INT markers, the bioinformatics part of studies and drafted the manuscript. Vinokourova S. carried out the APOT experiments and sequencing. Moisjak E. participated in the RH-mapping. Rakhmanaliev E. participated in the bioinformatics part of studies. Kobseva V. carried out the sequencing of the integration sites. Laimins L. participated in design of the study and writing of manuscript. Kisseljov F. and Sulimova G. conceived of the study, participated in design of the study and writing of manuscript.

All authors read and approved the final manuscript.

\section{Additional material}

\section{Additional File 1}

Homologies of INT markers and adjacent cellular sequences with human ESTs

Click here for file

[http://www.biomedcentral.com/content/supplementary/14712407-2-24-S1.doc]

\section{Additional File 2}

Physical locations of INT markers on human chromosome RH-maps Click here for file

[http://www.biomedcentral.com/content/supplementary/14712407-2-24-S2.doc]

\section{Acknowledgements}

This work was supported by Russian Human Genome Project (Grant 89'99), Russian Foundation of Basic Science (Grants 00-15-97777, 99-0448I9I, 02-04-4827I) and FIRCA Grant from NIH (0600-070-C223).

\section{References}

I. De Villiers E: Human pathogenic papillomavirus types: an upgrade. Curr Top Microbiol Immunol 1994, I 86: I- 12

2. Klaes R, Woerner S, Ridder R, Wentzetzen N, Duerst M, Schneider $A$, Lotz B, Melscheimer P, von Knebel Doeberitz M: Detection of high-risk cervical intraepithelial neoplasia and cervical cancer by amplification of transcripts derived from integrated papillomavirus oncogenes. Cancer Research 1999, 59:6 I 32-6I36

3. Kiselev F, Kiseleva N, Kobzeva V Gritsko T, Semenova L, Pavlova L, Klaes R, von Knebel Doeberitz M: Status of the human DNA papillomavirus in cervical tumors. Mol Biol (Mosk) 200I, 35:470-476

4. Van Tine B, Knops J, Broker T, Chow L, Moen P Jr: In situ analysis of the transcriptional activity of integrated viral DNA using tyramide-FISH. Dev Biol (Basel) 200I, 106:38I-385

5. Koopman L, Szuhai K, van Eendenburg J, Bezrookove V, Kenter G, Schuuring E, Tanke H, Fleuren G: Recurent integration of human papillomavirus 16, 45 and 67 near translocation breakpoints in new cervical cancer cell lines. Cancer Research 1999, 59:56I55624

6. Sastre-Garau X, Schneider-Maunoury S, Couturier J, Orth G: Human papillomavirus type I 6 DNA is integrated into chromosome region 12 q $14-q \mid 5$ in a cell line derived from a vulvar intraepithelial neoplasia. Cancer Genet Cytogenet I 990, 44:243-25 I

7. Gilles C, Piette J, Ploton D, Doco-Fenzy M, Foidart J: Viral integration sites in human papilloma virus-33-immortalized cervical keratinocyte cell lines. Cancer Genet Cytogenet 1996, 90:63-69

8. Wilke C, Hall B, Hoge A, Paradee W, Smith D, Glover T: FRA3B extends over a broad region and contains a spontaneous HPV I 6 integration site: direct evidence for the coincidence of viral integration sites and fragile sites. Hum Mol Genet 1996, 5:187-195

9. Cannizzaro L, Durst M, Mendez M, Hecht B, Hecht F: Regional chromosome localization of human papillomavirus integration sites near fragile sites, oncogenes, and cancer chromosome breakpoints. Cancer Genet Cytogenet 1988, 33:93-98

10. el Awady M, Kaplan J, O'Brien S, Burk R: Molecular analisis of integrated human papillomavirus 16 sequences in the cervical cancer cell lines SiHa. Virology 1987, I 59:389-398

II. Thorland E, Myers S, Persing D, Sarkar G, McGovern RM, Gostout B, Smith DI: Human papillomavirus type 16 integrations in cervical tumors frequently occur in common fragile sites. Cancer Res 2000, 60:5916-5921

12. Wentzensen N, Ridder R, Klaes R, Vinokurova S, Schaefer U, von Knebel Doeberitz M: Characterization of viral-cellular fusion transcripts in a large series of HPV 16 and 18 positive anogenital lesions. Oncogene 2002, 2 I:419-426

13. Samoylova E, Shaikhaiev G, Petrov S, Kisseljova N, Kisseljov F: HPV infection in cervical-cancer cases in Russia. Int J Cancer 1995, 6 I:337-341

14. Frohman M: Rapid amplification of complementary DNA ends for generation of full-length complementary DNAs: thermal RACE. Methods Enzymol 1993, 2 1 8:340-356

15. Sulimova GE, Kompaniitsev AA, Kunizheva SS, Klimov EA, Rakhmanaliev ER, Udina IG: Mapping of EST- and STS-markers in the human genome using a panel of radiation hybrids. Genetika 2000, 36:900-907

16. Udina IG, Baranova AV, Kompaniitsev AA, Sulimova GE: Evolutionarily-conserved gene CKAP2, located in region $13 \mathrm{q} / 4.3$ of the human genome, is frequently rearranged in various tumors. Genetika 200I, 37:120-123

17. Davis D, Delmonte A, Ly C, McNally E: Myoferlin, a candidate gene and potential modifier of muscular dystrophy. Hum Molec Genet 2000, 9:217-226

18. Fernandez-Pol J, Klos D, Hamilton P: Metallopanstimulin gene product produced in a baculovirus expression system is a nuclear phosphoprotein that binds to DNA. Cell Growth Diff 1994, 5:8II-825

19. Suetsugu S, Miki H, Takenawa T: Identification of two human WAVEISCAR homologues as general actin regulatory mole- 
cules which associate with the Arp2/3 complex. Biochem Biophys Res Commun 1999, 260:296-302

20. Mock B, Kozak C, Seldin M, Ruff N, D'Hoostelaere L, Szpirer C, Levan $\mathrm{G}$, Seuanez H, O'Brien S, Banner C: A glutaminase (GLS) gene maps to mouse chromosome I, rat chromosome 9 and human chromosome 2. Genomics 1989, 5:291-297

21. Elgadi KM, Meguid RA, Qian M, Souba WW, Abcouwer SF: Cloning and analysis of unique human glutaminase isoforms generated by tissue-specific alternative splicing. Physiol Genomics 1999, 3I:5I-62

22. Peitsaro $P$, Johansson $B$, Syrjanen $S$ : Integrated human papillomavirus type 16 is frequently found in cervical cancer precursors as demonstrated by a novel quantitative real-time PCR technique. J Clin Microbiol 2002, 40:886-891

23. Watts K, Thompson C, Cossart Y, Rose B: Sequence variation and physical state of human papillomavirus type 16 cervical cancer isolates from Australia and New Caledonia. Int J Cancer 2002, 97:868-874

24. Vogelstein B, Kinzler K: p53 function and dysfunction. Cell 1992, 70:523-526

25. Hartwell L: Defects in a cell cycle checkpoint may be responsible for the genomic instability of cancer cells. Cell 1992, 71:543-546

26. zur Hausen $\mathrm{H}$ : Human papillomaviruses in the pathogenesis of anogenital cancer. Virology 1991, 184:9-13

\section{Pre-publication history}

The pre-publication history for this paper can be accessed here:

http://www.biomedcentral.com/1471-2407/2/24/prepub
Publish with BioMed Central and every scientist can read your work free of charge

"BioMedcentral will be the most significant development for disseminating the results of biomedical research in our lifetime."

$$
\text { Paul Nurse, Director-General, Imperial Cancer Research Fund }
$$

Publish with BMC and your research papers will be:

- available free of charge to the entire biomedical community

- peer reviewed and published immediately upon acceptance

- cited in PubMed and archived on PubMed Central

- yours - you keep the copyright

Submit your manuscript here:

http://www.biomedcentral.com/manuscript/
BioMedcentral.com editorial@biomedcentral.com 